\title{
USO DE SERIES SINTÉTICAS DE CAUDALES MENSUALES EN EL CÁLCULO DEL COSTO MARGINAL DE ENERGÍA DEL SISTEMA ELÉCTRICO INTERCONECTADO NACIONAL DEL PERÚ
}

\author{
Douglas Sarango J. ${ }^{\mathrm{a}^{*}}$, Teresa Velásquez B. ${ }^{\mathrm{b}}$ \\ ${ }^{a}$ Departamento Académico de Ingeniería Mecánica de Fluidos, Facultad de Ciencias Físicas, Universidad Nacional Mayor \\ de San Marcos Ap. Postal 14-0149, Lima 14, Perú. \\ ${ }^{b}$ Departamento Académico de Recursos de Agua y Tierra, Facultad de Ingeniería Agrícola-UNALM.
}

(Recibido Junio 10, 2009; Aceptado 06, Setiembre 2009)

\begin{abstract}
Resumen
En este trabajo de investigación se evaluó la factibilidad de usar series sintéticas de caudales medios mensuales para la determinación de los costos marginales promedio de energía del Sistema Eléctrico Interconectado Nacional del Perú - SEIN, tomándose como caso de aplicación el estudio de la Fijación de Tarifas en Barra del año 2004, donde se usó el modelo de simulación, planificación y optimización del sistema energético y eléctrico peruano llamado modelo PERSEO. El modelo es usado actualmente por la Gerencia Adjunta de Regulación Tarifaría (GART) del Organismo de Supervisión de la Inversión en Energía y Minería OSINERGMIN.

El modelo usa como información hidrológica series de caudales medios mensuales de afluentes en los puntos de interés de las 23 cuencas hidrográficas del SEIN, siendo una de las principales la cuenca del lago Junín, cuyo recurso hídrico es usado por las centrales hidroeléctricas Mantaro y Restitución, generando casi el 20\% de la energía eléctrica de nuestro país.

Con el programa HEC-4, desarrollado por el Hydrological Engineering Center de los EE.UU. de América, a partir de la serie histórica de caudales mensuales históricos afluentes al lago Junín, se generaron 50 series de caudales mensuales sintéticos, determinándose a partir de ellas una serie seca, media y húmeda; información que se usó en el modelo PERSEO para calcular los costos marginales promedio de energía del SEIN para cada caso.

Los resultados obtenidos del costo marginal promedio de energía con el uso de series sintéticas de caudales mensuales, para el caso-seco, caso-medio y caso-húmedo, con el modelo PERSEO, al compararlos con el casohistórico, son menores en el orden de $1.14,1.55$ y 0.87 US \$/MWh, resultados que determinarán una disminución de los precios de la energía de los usuarios finales, como es el caso del usuario de tipo doméstico, comercial, industrial y minero.
\end{abstract}

Palabras claves: Series sintética de caudales mensuales, PERSEO, HEC-4, sistema eléctrico interconectado nacional, programación dinámica estocástica, centrales térmicas, centrales hidráulicas.

\begin{abstract}
In this research it was determined the feasibility of using synthetic series of monthly average flow for the determination of the average marginal cost of energy in the National Interconnected Electric System of Peru SEIN, taking as a case study of implementing bar tariff setting in 2004, where it was used the PERSEO model for planning, simulation and optimization of the hydrothermal system in Peru. The model is currently used by the Deputy Manager of tariff regulation (GART) of the Agency for Supervision of Investment in Energy and Mining - OSINERGMIN.

The model use as hydrological information the average monthly flow series of tributaries to the historical attractions of the 23 river basins of the SEIN, one of the main is the basin of the Junin Lake, whose water is used by the Mantaro and Restitution hydroelectric, generating almost $20 \%$ of the power of our country.

With the HEC-4 program, developed by the Hydrological Engineering Center of the USA, from the time series of monthly historical flows tributary to the Junin Lake, 50 series were generated synthetic monthly flow, determined from them a dry series, a average series and a wet series, information that was used in the PERSEO model to calculate the average marginal cost of energy of SEIN for each case.
\end{abstract}

\footnotetext{
* Correspondig author.e-mail: dsarangoj@unmsm.edu.pe
} 
The results obtained from the average marginal cost of energy with the use of synthetic series of monthly flows, for the dry case, average case and wet case, with the PERSEO model, compared to the historical event, are lower in order of $1.14,1.55$ and $0.87 \mathrm{US} \$ / \mathrm{MWh}$, the results will determine a decline in energy prices for end users, such as the domestic, commercial, industrial and mining users.

Keywords: Synthetic Series of monthly flow, PERSEO, HEC-4, National Interconnected Electric System, stochastic dynamic programming, power stations, hydro.

\section{Introducción}

Nuestro país cuenta con el Sistema Eléctrico Interconectado Nacional -SEIN, el cual transfiere la energía desde las centrales de generación hidráulica y térmica hacia los centros de demanda, por lo tanto cubre la demanda máxima de potencia de nuestro país, que en el año 2004 fue de 3092 MW, demanda que corresponde a la zona costera, desde la localidad de Mancora-departamento de Ica- por el sur hasta el departamento de Tumbes por el norte, y desde la ciudad de Ayacucho hasta la ciudad de Tingo María en la zona central del país. Mientras que por el sur desde Quillabamba en el Cuzco hasta Ilave en Puno, y desde Arequipa hasta Tacna por el sur (Fig. 1).

El Organismo de Supervisión de la Inversión en Energía y Minería (OSINERGMIN) por mandato de la Ley de Concesiones Eléctricas - D.L. No. 25844 [1], debe garantizar la seguridad de la generación eléctrica para cubrir la demanda eléctrica de nuestro país en el corto y mediano plazo, para lo cual efectúa el planeamiento de la optimización de la expansión de la generación del Sistema Eléctrico Interconectado Nacional (SEIN) para optimizar los recursos hidráulicos y térmicos existentes y futuros usando el modelo de simulación, planificación y optimización del sistema energético y eléctrico peruano - PERSEO [2].

El SEIN está conformado por centrales hidráulicas y centrales térmicas, líneas de transmisión, subestaciones eléctricas, sistemas de compensación reactiva y cargas, que transfieren la energía desde los centrales de generación hidráulica y térmica hacia los centros de demanda.

Las empresas de generación eléctrica del SEIN venden la energía eléctrica que generan en sus centrales hidroeléctricas y térmicas a un costo denominado Costo Marginal de Energía (CME) [3], que es obtenido con el modelo PERSEO.

Actualmente la energía eléctrica generada en el SEIN es $60 \%$ generación hidráulica y el $40 \%$ generación térmica, donde los combustibles usados por las centrales térmicas son gas natural de Camisea, Petróleo Diesel-2, Petróleo Residual 500, Petróleo Residual 5, Petróleo Residual 6 y Carbón, mientras que la generación hidroeléctrica es a base del recursos hídrico proveniente de 23 cuencas hidrográficas.

El PERSEO para su componente hidráulicahidrológica utiliza información de series de caudales medios mensuales naturales afluentes a los embalses y tomas que forman parte de los sistemas hidráulicos asociados a las centrales hidroeléctricas del SEIN.

En este sentido el CME del SEIN es calculado considerando la generación hidroeléctrica obtenida en función de la optimización de la operación de los embalses del sistema utilizando las series de caudales mensuales naturales afluentes históricos.

En el presente trabajo se ha considerado que la cuenca hidrográfica más importante del SEIN es la del río Mantaro, específicamente el embalse Lago Junín, (Fig. 2). En el PERSEO se usa sólo la serie histórica de caudales mensuales afluentes al lago Junín, asumiendo que ésta se va a presentar tan igual en el futuro, pero se sabe que existe la posibilidad de que esto no ocurra, por lo tanto es probable que se presenten otras series de caudales equiprobables de ingreso al lago y que podrían ser usadas en el modelo. En este sentido con el modelo HEC-4 [4], [5], se han generado 50 series sintéticas de caudales mensuales equiprobables de entrada al embalse Junín, de igual longitud que la serie histórica de ingreso, éstas series que mantienen los parámetros estadísticos de la serie histórica, como es la media y la desviación estándar.

A partir de las series sintéticas generadas, se determinó una serie seca, promedio y húmeda. Con esta información en el PERSEO se han calculado el costo marginal de energía del SEIN para cada uno de las series hidrológicas.

Los resultados obtenidos con el PERSEO usando las series de caudales sintéticas, indican que los costos marginales de energía del SEIN, los volúmenes útiles del lago Junín y la producción de energía en las centrales hidroeléctricas Mantaro y Restitución son diferentes a los obtenidos considerando la serie histórica de ingreso al lago Junín, lo cual se traducirá en un costo menor de energía en el usuario final, como es el caso del usuario de tipo doméstico.

\section{Formulación de la Hipótesis}

La generación de series sintéticas de caudales medios mensuales en las cuencas del Sistema Eléctrico Interconectado Nacional del Perú (SEIN) es una opción para el cálculo de los costos marginales de energía usando el modelo PERSEO, lo cual mejoraría la determinación de los costos de energía del usuario final de tipo doméstico 


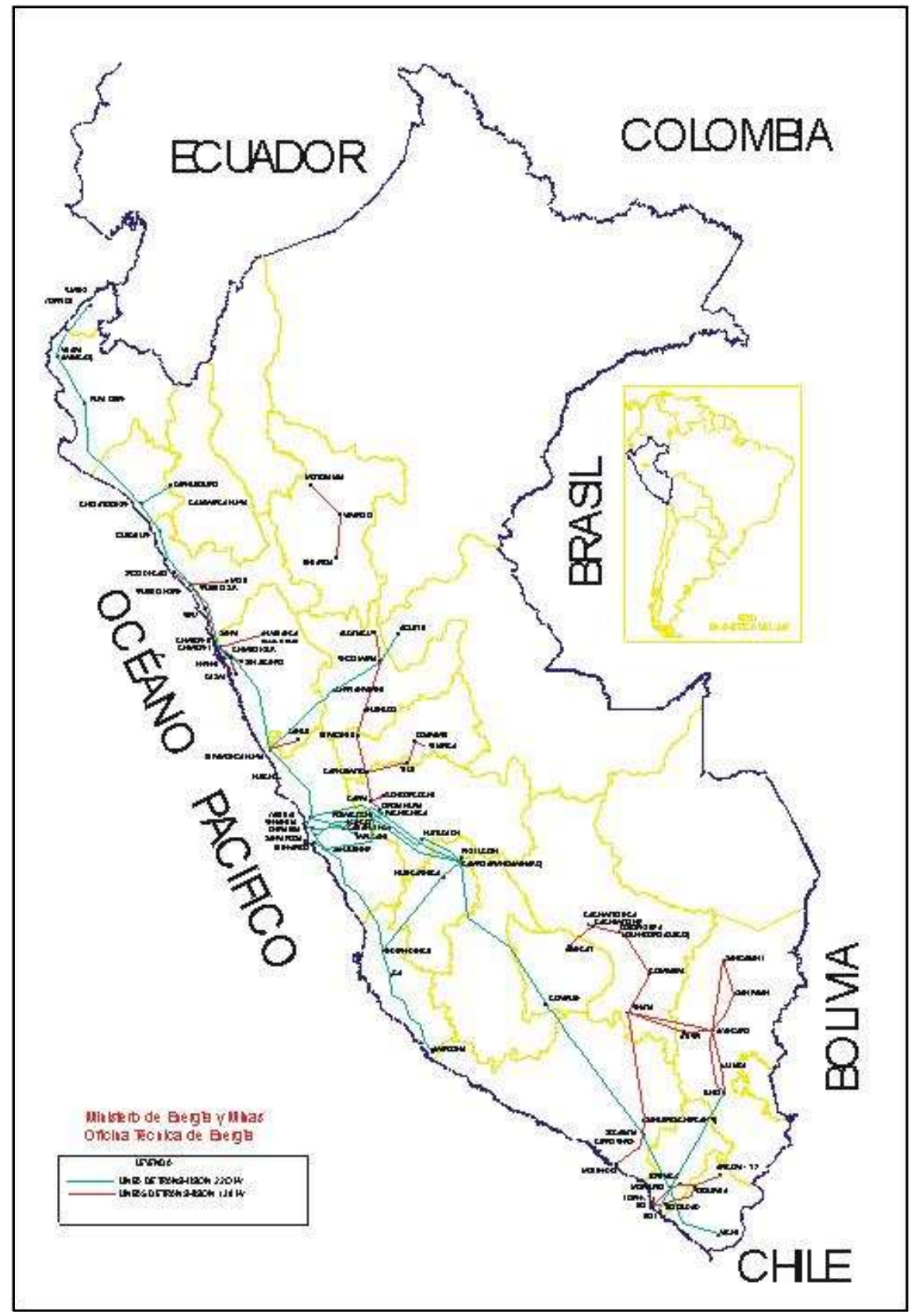

Fig. 1. Sistema Eléctrico Interconectado Nacional - SEIN

\section{Materiales y Métodos}

\subsection{Modelo PERSEO}

El Sistema Eléctrico Interconectado Nacional (SEIN), es un sistema hidrotérmico, los suministradores de energía eléctrica son las plantas hidroeléctricas y termoeléctricas. El consumidor se representa por la demanda total del mercado eléctrico. Las plantas hidroeléctricas turbinan el agua regulada por uno o mas embalses, dispuestos o no en cascada, a lo largo de una o más cuencas hidrográficas, lo cual permite distribuir óptimamente la energía para usar en diferentes periodos.

Las características más importantes en la operación de los sistemas hidrotérmicos la constituye el manejo de la energía hídrica almacenada en los embalses del sistema, evitando así los gastos de combustible que ocasionaría la generación térmica. En otras palabras, si se utilizan las reservas del agua para evitar costos por generación térmica en la actualidad, y en el futuro se presenten caudales 
afluentes bajos, es decir, ocurriría una sequía, elevando los costos. Si, por otro lado, se decide almacenar agua incurriendo en un mayor uso de generación térmica en la actualidad y ocurriesen en el futuro unos caudales afluentes elevados, ocurrirían vertimientos en los embalses con el consiguiente desperdicio de energía, ocasionando un aumento de los costos operativos.

Las ecuaciones de conservación del agua dentro del modelo PERSEO, representan el balance hídrico en cada punto de las cuencas hidrográficas, es así como el volumen final del embalse en la etapa $t$ es igual al volumen inicial (final de la etapa anterior), más las entradas de agua (caudales incrementales afluentes y caudales provenientes de las plantas aguas arriba), menos las pérdidas por evaporación, y los volúmenes turbinados y vertidos en el propio embalse [2], [6], [7].

$$
\begin{aligned}
& v_{i, t+1=} v_{i, t}+a_{i, t+1}+\sum_{l \varepsilon M i}\left(q_{l}+s_{l}\right)-q_{i}-s_{i}-e_{i, t+1} \\
& e_{i, t+1=} \varepsilon v_{i, t}
\end{aligned}
$$



Donde:

$v_{i, t} \quad$ Volumen de agua almacenado en el embalse de la i-ésima planta al inicio de la etapa $t$ (igual al volumen almacenado en la etapa $t-1$ )

$a_{i, t} \quad$ Volumen de agua afluente al embalse de la iésima planta durante la etapa $t$

$S_{i, t} \quad$ Volumen de agua vertido por la i-ésima planta durante la etapa $t$

$M_{i}$ Conjunto de plantas aguas arriba de la i-ésima planta

$E_{i, t} \quad$ Volumen de agua evaporada en el embalse de la i-ésima planta en la etapa $t$ $\varepsilon \quad$ Porcentaje de evaporación respecto del volumen almacenado en el embalse.

Dentro de los sistemas hidráulicos asociados a las centrales hidroeléctricas, existen reservorios de regulación diaria, que le permiten almacenar agua en corto tiempo para ser usada en horas de máxima demanda, donde la central puede generar su máxima potencia. Se asume que estos reservorios son embalse pequeños que brindan regulación diaria, de modo que su operación esta referida solo a los bloque horarios que constituyen cada etapa, por lo que el volumen final del reservorio al igual que el inicial en la etapa $t$ es nulo. Dentro de la etapa el volumen del reservorio en el bloque $k$ es igual al volumen inicial (final del bloque posterior), más las entradas de agua (caudales incrementales afluentes y caudales provenientes de las plantas aguas arriba), menos los volúmenes turbinados y vertidos en el propio reservorio durante cada bloque horario. Debe quedar claro que la finalidad de incluir los reservorios dentro de la formulación es la de modelar con mayor detalle la disponibilidad de agua por parte de las centrales hidroeléctricas de acuerdo a los bloques horarios de demanda en cada etapa

$$
v_{i, t, k-1=} v_{i, t, k}+a_{i, t}+\sum_{l \varepsilon M i}\left(q_{l}+s_{l}\right)-q_{i}-s_{i, t, k-1}
$$


Donde:

$v_{i, t, k}$ Volumen de agua almacenado en el reservorio de iésima planta durante la etapa $t$ en el bloque $k$.

$a_{i, t} \quad$ Volumen de agua afluente al embalse de la i-ésima planta durante la etapa $t$

$S_{i, t, k}$ Volumen de agua vertido por la i-ésima planta durante el bloque $k$ de la etapa $t$

$M_{i} \quad$ Conjunto de plantas aguas arriba de la i-ésima planta. 
DIAGRAMA TOPOLOGICO DE LA CUENCA DEL RIO MANTARO
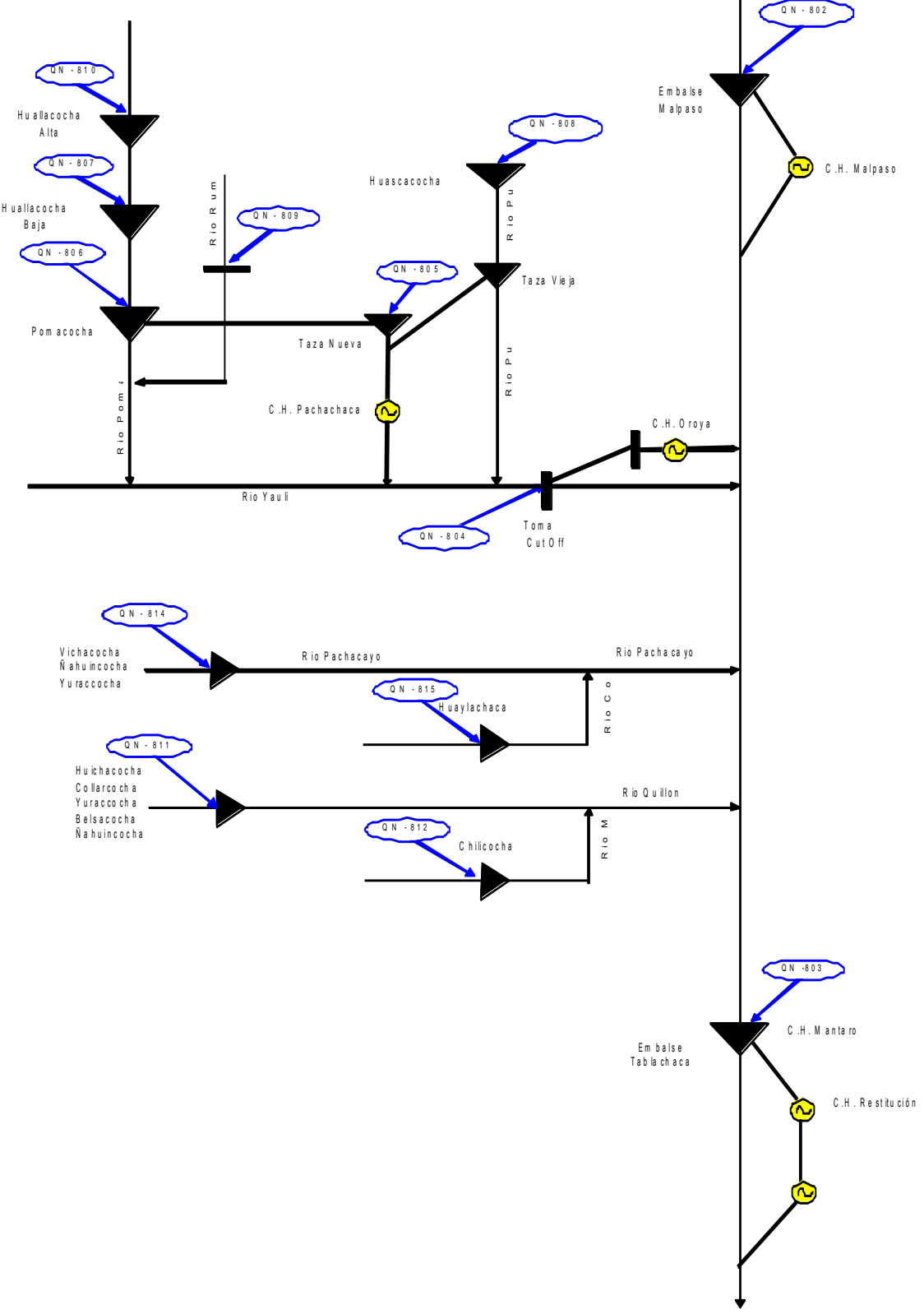

Fig. 2. Cuenca del río Mantaro en el SEIN

El modelo matemático de operación de sistemas hidrotérmicos para una secuencia hidrológica $s$, se puede representar a través del modelo recursivo en programación dinámica estocástica, como se indica en la siguiente ecuación: $\alpha_{t}\left(v_{t}, A_{t-1}\right)=\mathrm{E}_{A t / A t+1}\left\{\operatorname{Min}\left[\sum_{j=1}^{N j} c o_{j} g t_{j, t}+\alpha_{t+1}\left(v_{t+1}, A_{t}\right)\right]\right\}$

s/a 


$$
\begin{aligned}
& \sum_{j z k} g t_{j, t}+\sum_{i z k} \rho_{i} q_{i, j}+\sum_{m \varepsilon \Omega(k)}\left(\left(1-0.5 \gamma_{m k, t}\right) f_{m k, t}-\left(1+0.5 \gamma_{m k, t}\right) f_{m k, t}\right)=d_{k, t} \quad \pi_{d_{k, t, s}}^{c p} \\
& g t_{j, t} \leq \overline{g t}_{j, t} \\
& f_{m k, t} \leq \bar{f}_{m k} \\
& v_{i, t+1}-v_{i, t}-\sum_{l \varepsilon M_{i}}\left(q_{l}+s_{l}\right)+q_{i}+s_{i}=a_{i, t}-e_{i, t} \\
& v_{i, t, k-1}-v_{i, t, k}-\sum_{l \varepsilon M_{i}}\left(q_{l}+s_{l}\right)+q_{i}+s_{i}=a_{i, t} \\
& v_{i, t} \leq \bar{v}_{i, t} \\
& v_{i, t} \geq \underline{v}_{i, t} \\
& v_{i, t, k} \leq \bar{v}_{i} \\
& q_{i, t} \leq \bar{q}_{i, t} \\
& q_{i, t} \geq \underline{q}_{i, t} \\
& r_{i, t} \leq \bar{r}_{i, t} \\
& r_{i, t} \geq \underline{r}_{i, t} \\
& f_{m k, t}=f_{m k}\left(\theta_{m}, \theta_{k}\right) \geq 0 \\
& \forall k=1, \ldots . ., N_{k} ; i=1, \ldots ., N_{I} ; j=1, \ldots ., N_{j} ; t=1, \ldots ., N_{t} \\
& \pi_{g t_{j, t, s}}^{c p} \\
& \pi_{f_{m k, t, s}}^{c p} \\
& \pi_{a_{i, t, s}}^{c p} \\
& \pi_{v_{i, t, s}}^{c p} \\
& \pi_{q_{i, t, s}^{c p}}^{c p} \\
& \pi_{r_{i, t, s}}^{c p}
\end{aligned}
$$

Donde:

$\mathrm{K} \quad$ índice de las barras equivalentes

$N_{k} \quad$ Número total de barras equivalentes en el sistema

$\Omega_{k} \quad$ Conjunto de líneas de transmisión conectadas a la barra equivalente $k$

$f_{m k, t} \quad$ Flujo de energía de la barra equivalente $m$ para la barra equivalente $k$ en la etapa $t$

$\gamma_{m k, t} \quad$ Coeficiente de pérdidas de flujo de transmisión de m para $k$ durante la etapa $t$, expresado en $\%$ del flujo actual

$d_{k, t} \quad$ Demanda de energía en la barra equivalente $k$ durante la etapa $t$

$\bar{v}_{i, t} \quad$ Volumen del embalse mínimo

$\underline{v}_{i, t} \quad$ Volumen del embalse máximo

$\bar{v}_{i} \quad$ Volumen máximo en los reservorios diarios

$q_{i, t} \quad$ Volumen turbinado en la i-ésima planta hidroeléctrica durante la etapa $t$

$\bar{q}_{i, t} \quad$ Volumen turbinado máximo

$\underline{q}_{i, t} \quad$ Volumen turbinado mínimo

$r_{i, t} \quad$ Caudales regulados

$\underline{r}_{i, t} \quad$ Caudales regulados mínimos de irrigación, navegación o recreación

$\bar{r}_{i, t} \quad$ Caudales regulados máximos caso de grandes avenidas

\subsection{Modelo HEC-4}

Este programa fue implementado por el Hydrologic Engineering Center of USA [3], analiza caudales mensuales en un número de estaciones interrelacionadas para determinar sus características estadísticas y generar una secuencia de caudales hipotéticos de cualquier longitud deseada que tenga esas características. Puede reconstruir caudales faltantes sobre la base de caudales concurrentes 
observados en otras localidades para obtener cantidades máximas y mínimas en cada mes y para cada duración especifica en los caudales registrados, reconstituidos y generados. También usa el modelo de simulación generalizado para generar caudales mensuales en localizaciones que no cuenten con registros, en base a estudios regionales. El modelo HEC-4 tiene un uso más difundido en nuestro país que otros modelos existentes en el mercado.

\subsection{Información Utilizada en el modelo PERSEO}

a) Demanda Eléctrica

De los resultados obtenidos de la proyección de la demanda eléctrica son utilizados en el Sistema Eléctrico Interconectado Nacional (SEIN) elaborado por el OSINERG-GART [8], para el período 2004 2008, obtenidos para la fijación de precios en barra para el año 2004 (Tabla 1).

Tabla 1: Proyección Demanda SEIN -Período 2004-2008

\begin{tabular}{|c|c|c|c|c|c|}
\hline \multirow[b]{2}{*}{ Año } & \multirow{2}{*}{$\begin{array}{c}\text { Max. } \\
\text { Demanda } \\
(\mathrm{MW})\end{array}$} & \multirow{2}{*}{$\begin{array}{c}\text { Consumo } \\
\text { Anual }(\mathrm{GW} / \mathrm{h})\end{array}$} & \multirow{2}{*}{$\begin{array}{l}\text { Factor de Carga } \\
\qquad(\%)\end{array}$} & \multicolumn{2}{|c|}{ Tasa de Crecimiento } \\
\hline & & & & Potencia & Energía \\
\hline 2003 & 2971 & 20737 & $79.70 \%$ & & \\
\hline 2004 & 3092 & 21848 & $80.70 \%$ & $4.10 \%$ & $5.40 \%$ \\
\hline 2005 & 3269 & 22603 & $78.90 \%$ & $5.70 \%$ & $3.50 \%$ \\
\hline 2006 & 3407 & 23488 & $78.70 \%$ & $4.20 \%$ & $3.90 \%$ \\
\hline 2007 & 3562 & 24628 & $78.90 \%$ & $4.60 \%$ & $4.90 \%$ \\
\hline 2008 & 3699 & 25519 & $78.70 \%$ & $3.90 \%$ & $3.60 \%$ \\
\hline
\end{tabular}

b) Situación del Sistema Eléctrico Interconectado Nacional (SEIN)

La situación del SEIN al año 2003 [8], está representada por las características técnicas y económicas de las centrales hidráulicas y térmicas existentes en ese año. En la Tabla 2 se presenta las empresas generadoras de electricidad y su generación hidroeléctrica en el año 2003. De igual manera en la Tabla 3, se muestra la generación térmica de cada una de las empresas del SEIN al 2003.

Tabla 2: Generación Hidráulica del SEIN - 2003

\begin{tabular}{lcc}
\hline \multicolumn{1}{c}{ Empresa } & $\begin{array}{c}\text { Potencia } \\
\text { Efectiva } \\
(\mathrm{Mw} .)\end{array}$ & $\begin{array}{c}\text { Energía Media } \\
\text { Anual } \\
(\mathrm{GW} / \mathrm{h})\end{array}$ \\
\hline EGE CAHUA & 52.8 & 419.5 \\
DEI EGENOR & 358.5 & 2249.2 \\
ELECTROPERU & 851.0 & 7022.7 \\
EDEGEL & 759.0 & 4709.8 \\
ELECTROANDES & 170.6 & 1233.1 \\
ENERGIA & 38.1 & 172.5 \\
PACASMAYO & 27.9 & 146.2 \\
SINERSA & 170.2 & 771.5 \\
EGASA & 34.9 & 130.7 \\
EGESUR & 86.8 & 744.2 \\
EGEMSA & 113.1 & 783.0 \\
SAN GABAN & & \\
\hline
\end{tabular}

Fuente: OSINERG-GART

\section{c) Programa de Obras}

El programa de obras está dado por la secuencia de equipamiento de generación y transmisión esperado para ingresar al servicio dentro del periodo de análisis de 48 meses (2004-2008) señalado por la Ley de Concesiones Eléctricas (LCE). Para establecer el programa de obras se tiene en cuenta aquellas factibles de entrar en operación en el periodo, considerando las que se encuentran en construcción y aquellas contempladas en el Plan Referencial de Electricidad elaborado por el Ministerio de Energía y Minas (MEMIN) el 2003 [9], entre otras. El programa de obras de generación del SEIN a emplearse se muestra en la Tabla 4, respectivamente.

Tabla 3: Generación Térmica del SEIN - 2003

\begin{tabular}{lc}
\hline \multicolumn{1}{c}{ Empresa } & $\begin{array}{c}\text { Potencia Efectiva } \\
\text { (Mw.) }\end{array}$ \\
\hline EEPSA & 125.0 \\
DEI EGENOR & 183.3 \\
ENERGIA PACASMAYO & 24.6 \\
EDEGEL & 227.1 \\
TRUPAL & 13.9 \\
SHOUGESA & 77.6 \\
TERMOSELVA & 165.1 \\
ELECTROPERU & 18.2 \\
ELECTROUCAYALI & 23.8 \\
ETEVENSA & 324.6 \\
EGEMSA & 11.8 \\
SAN GABAN & 10.3 \\
EGASA & 148.4 \\
EGESUR & 26.1 \\
ENESUR & 360.0 \\
\hline Fuente: OSINERG-GART &
\end{tabular}


Tabla 4: Proyectos de Generación en el SEIN Período 2004-2008

\begin{tabular}{|c|c|}
\hline $\begin{array}{l}\text { Fecha de } \\
\text { Ingreso }\end{array}$ & Proyecto \\
\hline Enero 2004 & Presa Pillones (71 MMC) \\
\hline Enero 2005 & $\begin{array}{l}\text { Regulación de Laguna Rajucolta } \\
\text { (10 MMC) }\end{array}$ \\
\hline Abril 2005 & $\begin{array}{l}\text { Rehabilitación del grupo } 1 \mathrm{CH} \\
\text { Callahuanca ( } 2.5 \mathrm{MW})\end{array}$ \\
\hline Julio 2005 & $\begin{array}{l}\text { Rehabilitación del grupo } 2 \mathrm{CH} \\
\text { Callahuanca }(2.5 \mathrm{MW})\end{array}$ \\
\hline Julio 2005 & CH Yuncán (130 MW) \\
\hline Octubre 2005 & $\begin{array}{l}\text { Rehabilitación del grupo } 3 \mathrm{CH} \\
\text { Callahuanca ( } 2.5 \mathrm{MW})\end{array}$ \\
\hline Junio 2006 & $\begin{array}{l}\text { TGN Ciclo combinado } 225 \mathrm{MW} \\
\text { (Reconversión Ventanilla TG 4) }\end{array}$ \\
\hline
\end{tabular}

d) Otra información considerada

Además de la información indicada en los ítems a), b) y c), también se obtuvo información referente a las: barras del SEIN, líneas de transmisión, combustibles, empresas que agrupan centrales termoeléctricas e hidroeléctricas, grupos termoeléctricos, cuencas hidrográficas, bocatomas existentes en las cuencas, embalses y reservorios diarios existentes, centrales hidroeléctricas existentes, configuración hídrica de las cuencas, series de caudales medios mensuales afluentes, valores de requerimientos de agua, valores de demanda de energía eléctrica en barra, horas de mantenimiento en punta y fuera de punta por año y mes de los grupos termoeléctricos e hidroeléctricos del SEIN [8].

\subsection{Metodología}

Con la información recabada en el ítem 3.2, se siguió la metodología de trabajo siguiente:

a) Descripción del Sistema Eléctrico Interconectado Nacional (SEIN) al año 2003.

b) Se elaboraron los archivos de datos del modelo PERSEO, considerando la serie de caudales mensuales históricos de ingreso al lago Junín (Caso-Hist) para el cálculo de los costos marginales promedio de energía del SEIN, período 2004-2008.

c) Se elaboro el archivo de datos del modelo HEC4, para la generación de series sintéticas de caudales mensuales de ingreso al Lago Junín.

d) Se generó 50 series sintéticas de caudales mensuales de la misma longitud de la serie histórica (1965-2003), 39 años cada una. e) Se determinó a partir de las series sintéticas de caudales mensuales generadas, la serie seca (Caso-S), serie promedio (Caso-M) y serie húmeda (Caso-Hum).

f) Considerando como serie de ingreso al lago Junín, la serie seca, media y húmeda, y manteniendo los demás archivos de datos del PERSEO, se calculó los costos marginales de energía del SEIN, período 2004-2008, así como las demás características técnicas y económicas del sistema hidrotérmico.

\section{Resultados y Discusión}

\subsection{Costos marginales actualizados de energía del SEIN}

El costo promedio de los CM de energía actualizados para el período de estudio 2004-2008, para el Caso-Hist, Caso-S, Caso-M y Caso-Hum, son de $30.22,29.08,28.67$ y 29.35 US $\$ / M W h$, respectivamente. Estos resultados se exponen en la Tabla 5, de donde se deduce que los resultados obtenidos de los casos estudiados son menores al compararlos con el Caso-Hist, siendo estas diferencias del orden de 1.14, 1.55 y 0.87 US\$/MWh. En la Fig. 3, se presenta la comparación de los CMA a nivel mensual del período 2004-2008, de los casos antes mencionados.

Tabla 5: Costo Marginal Actualizado (US\$/MWh) en el SEIN: Período 2004-2008

\begin{tabular}{ccccc}
\hline Año & Caso-Hist & Caso-S & Caso-M & Caso-Hum \\
\hline 2004 & 27.72 & 26.59 & 26.41 & 26.91 \\
2005 & 28.44 & 27.33 & 27.02 & 27.61 \\
2006 & 31.34 & 30.24 & 29.73 & 30.47 \\
2007 & 33.37 & 32.19 & 31.53 & 32.43 \\
Prom. & 30.22 & 29.08 & 28.67 & 29.35 \\
\hline
\end{tabular}

Fuente: Elaboración propia con resultados del modelo Perseo

\subsection{Volumen útil del lago Junín}

Respecto al volumen útil del lago Junín en el período 2004-2008, el promedio es del orden de 215.59, 212.21, 207.08 y 219.52 MMC, para el CasoHist, Caso-S, Caso-M y Caso-Hum. El volumen útil promedio es menor para el Caso-S y Caso-M con respecto al Caso-Hist, siendo estos valores de 3.39 y 8.51 MMC, mientras que en el Caso-Hum es mayor en 3.92 MMC (Tabla 6). En la Fig. 4 se muestra la comparación del volumen útil del lago Junín a nivel mensual y se puede apreciar que no existe mayor deferencia en la tendencia de la trayectoria de embalse y desembalse del lago. 
Tabla 6: Volumen útil en lago Junín: Período 2004-2008

\begin{tabular}{ccccc}
\hline Año & Caso-Hist & Caso-S & Caso-M & Caso-Hum \\
\hline 2004 & 227.66 & 226.25 & 226.33 & 230.88 \\
2005 & 207.67 & 205.40 & 196.46 & 213.97 \\
2006 & 211.76 & 204.67 & 197.37 & 214.29 \\
2007 & 215.30 & 212.51 & 208.17 & 218.92 \\
Prom. & 215.59 & 212.21 & 207.08 & 219.52 \\
\hline
\end{tabular}

Fuente: Elaboración propia con resultados del modelo Perseo

\subsection{Energía mensual generada en las centrales de Mantaro y Restitución}

El promedio de la energía generada por las centrales hidroeléctricas Mantaro y Restitución, en el período 2004-2008, para el caso histórico es de 603.92 GWh, mientras que para el Caso-Hist, Caso$\mathrm{S}$, Caso-M y Caso-Hum la generación de energía alcanza los valores de $610.22,611.72$ y $608.52 \mathrm{GWh}$, respectivamente, observándose que en todos los casos es mayor que el Caso-Hist, ver Tabla 7. En la Fig. 5, se puede observar la comparación gráfica de la energía generada a nivel mensual en las $\mathrm{CCHH}$ Mantaro y Restitución en el período 2004-2008.

Tabla 7: Energía generada promedia CCHH Mantaro y Restitución: Período 2004-2008

\begin{tabular}{ccccc}
\hline Año & Caso-Hist & Caso-S & Caso-M & Caso-Hum \\
\hline 2004 & 618.07 & 624.02 & 625.59 & 622.64 \\
2005 & 596.20 & 602.23 & 603.54 & 600.45 \\
2006 & 595.18 & 602.88 & 604.68 & 600.49 \\
2007 & 606.25 & 611.76 & 613.07 & 610.52 \\
Prom. & 603.92 & 610.22 & 611.72 & 608.52 \\
\hline
\end{tabular}

Fuente: Elaboración propia con resultados del modelo Perseo

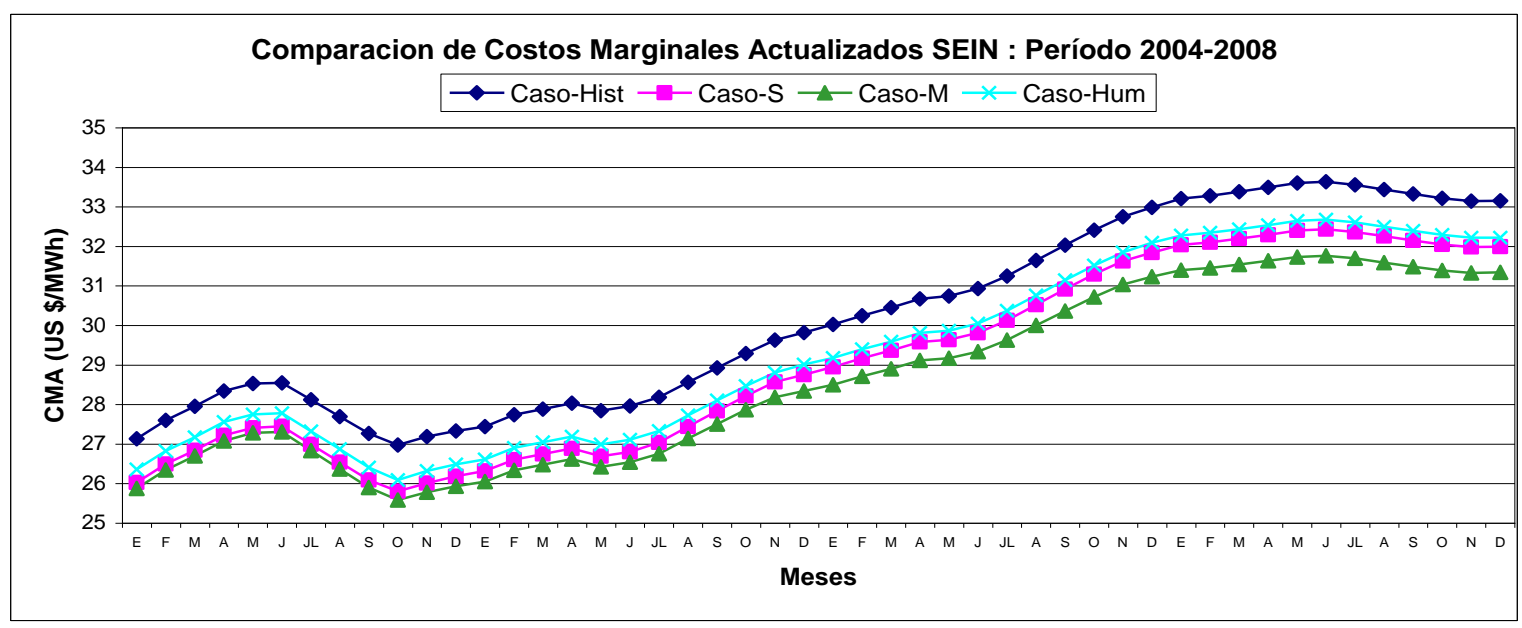

Fuente: Elaboración propia con resultados del modelo Perseo

Fig. 3. Comparación de costos marginales actualizados promedio del SEIN.

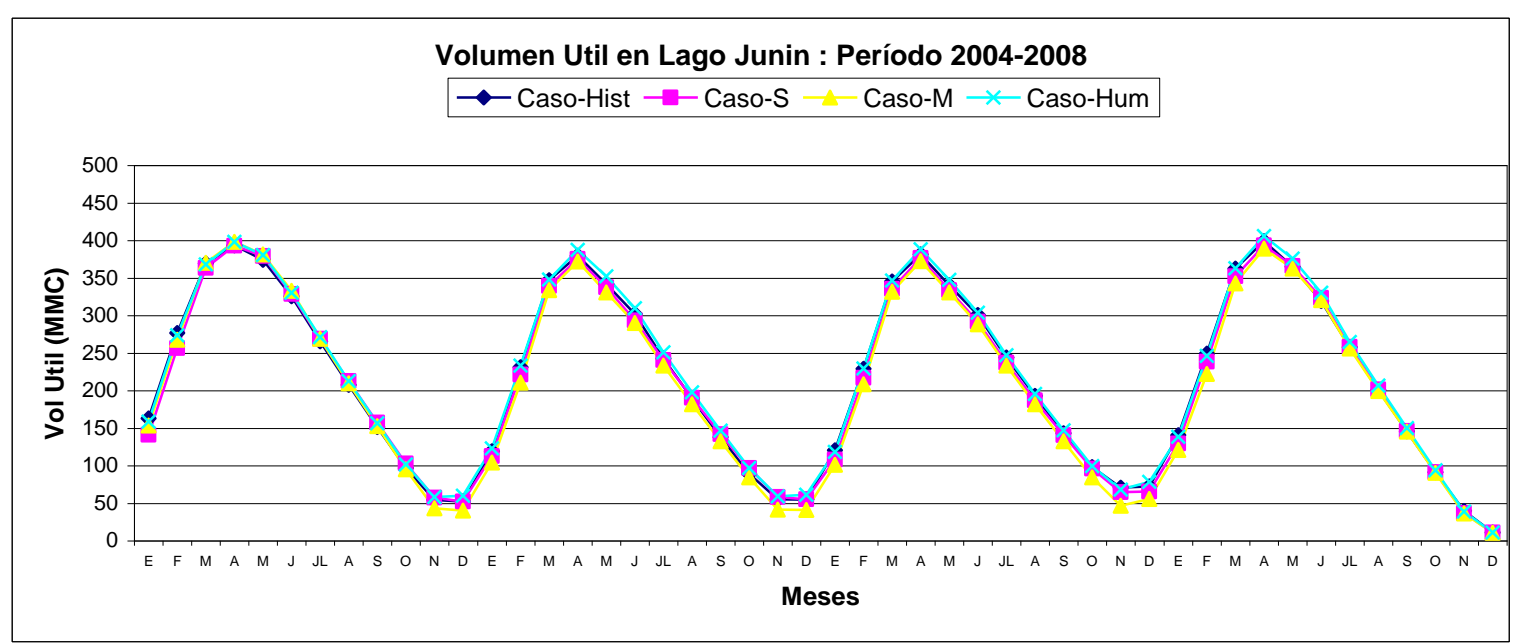

Fuente: Elaboración propia con resultados del modelo Perseo

Fig. 4. Comparación de volumen útil lago Junín en el SEIN. 


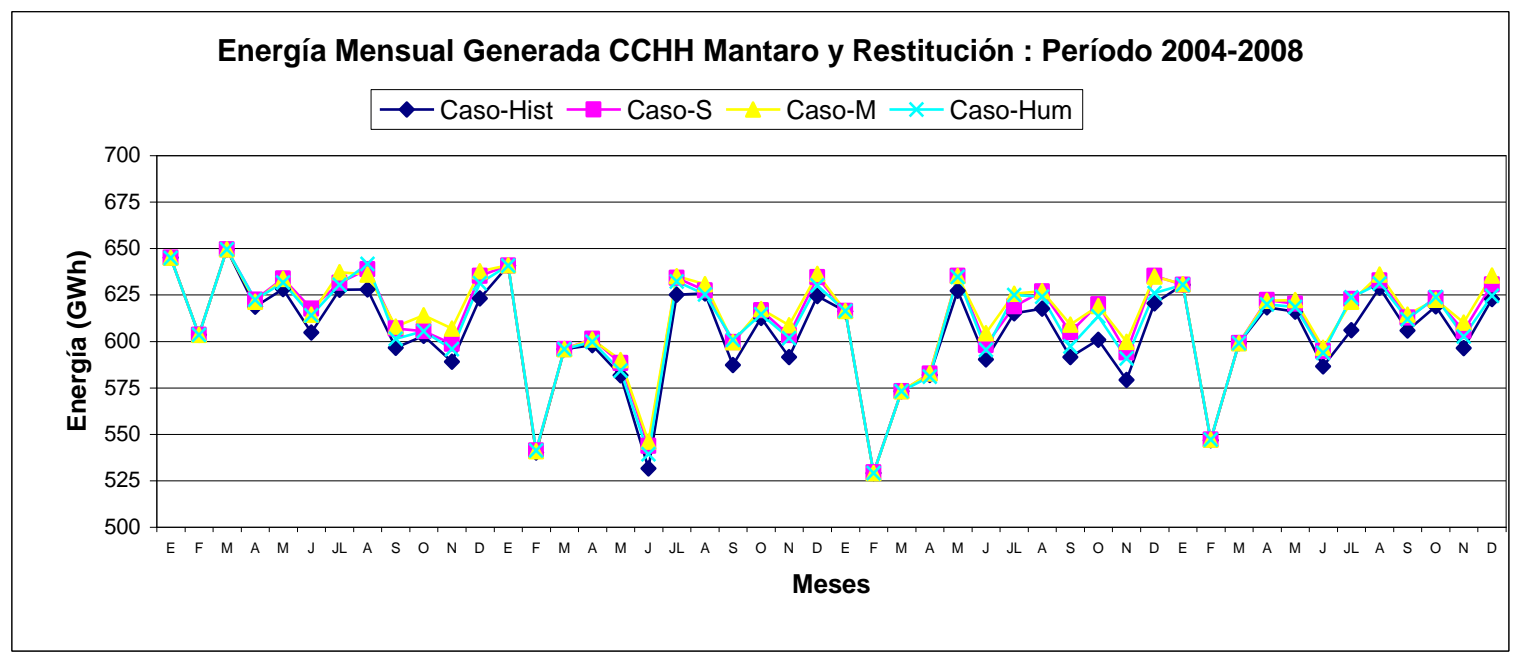

Fuente: Elaboración propia con resultados del modelo Perseo.

Fig. 5. Comparación energía generada en la CCHH Mantaro y Restitución.

\section{Conclusiones}

a) El modelo PERSEO utiliza series de caudales mensuales históricas afluentes a los puntos de interés de las cuencas hidrográficas del Sistema Eléctrico Interconectado Nacional del Perú (SEIN), como es el caso de la cuenca del lago JUNIN.

b) Los costos marginales de energía del SEIN se calculan con el modelo PERSEO, considerando la información referente a líneas de transmisión, combustibles, empresas que agrupan centrales termoeléctricas e hidroeléctricas, grupos termoeléctricos, cuencas hidrográficas, bocatomas existentes en las cuencas, embalses y reservorios diarios existentes, centrales hidroeléctricas existentes, configuración hídrica de las cuencas, series de caudales medios mensuales afluentes a los puntos de interés de las cuencas hidrográficas del sistema, valores de requerimientos de agua, valores de demanda de energía eléctrica en barra, horas de mantenimiento en punta y fuera de punta por año y mes de salida de los grupos termoeléctricos e hidroeléctricos del SEIN .

c) Es factible el uso de series sintéticas de caudales mensuales afluentes al lago Chinchaycocha del Dpto. de JUNIN, y pueden ser usadas en el modelo PERSEO para el cálculo de los costos marginales de energía del SEIN, lo que se traducirá en mejorar los costos de energía de los usuarios finales, como es el caso del usuario de tipo doméstico

\section{Referencias}

[1]. Decreto Ley $\mathrm{N}^{\circ} 25844$, Ley de Concesiones Eléctricas (LCE), noviembre 1992 y sus modificatorias. Reglamento de la Ley de Concesiones Eléctricas, aprobado por Decreto Supremo No. 009-93-EM, febrero 1993 y sus modificatorias.

[2]. Organismo Superior de la Inversión en Energía y Minería (OSINERGMIN), "Modelo de Simulación de la Operación Económica Optima del Sistema Peruano - PERSEO, Manual del Usuario," 2002.

[3]. Kamiyama A., Combe M. y Sarango D.,"Perfeccionamiento del Modelo de Cálculo de Costos Marginales SISPERU" - Comisión de Tarifas Eléctricas, 1996

[4]. Hydrology Engineering Center, USA, "HEC-4, Monthly Streamflow Simulation", 1970,

[5]. Tejada-Guilbert, J.A., Johnson, S.A., Stedinger, J.R., "The value of hydrologic information in stochastic dynamic programming models of a multireservoir system", Water Resources Research, Vol. 31, pp 2571-2579. 1995.

[6]. Pereira, M.V.F., Campodónico, N.M., Gorestin, B.G., Costa, J.P., "Application of Stochastic Optimization to Power System Planning and Operation", IEEE/KTH Stockholm Power Tech Conference, Stockholm, Sweden, JuneJune1822, 1995.

[7]. Pereira, M.V.F., Pinto, L.M.V.G., "Stochastic Optimization of a Multireservoir Hydroelectric System: A decomposition Approach", Water resources research, Vol. 21, No. 6, pp 779-792, June 1985,

[8]. Organismo Superior de la Inversión en Energía y Minería (OSINERGMIN), "Proceso de Fijación Tarifas en Barra del Costo Marginal 
Promedio de la Energía del Sistema Eléctrico Interconectado Nacional - SINAC, Abril 2003". [9]. Ministerio de Energía y Minas, "Plan
Referencial de Electricidad del Perú 20032014", Dirección General de Electricidad, Lima-Perú, 2003. 